\title{
Multicycle Investigation of Normal Paraffin Separation from Naphtha to Improve Olefin and Aromatic Feed
}

Jichang Liu*, Xiang Chen, Shimin Zhao, Xin Cao, Benxian Shen

State Key Laboratory of Chemical Engineering, East China University of

Science and Technology, Shanghai 200237, China

* Author to whom all correspondence should be addressed. E-mail: liujc@ecust.edu.cn (J.C. Liu)

Table S1. N-paraffin contents of SGPC naphtha

\begin{tabular}{lrrrrrrrr}
\hline n-paraffins & $\mathrm{n}-C_{4}^{0}$ & $\mathrm{n}-C_{5}^{0}$ & $\mathrm{n}-C_{6}^{0}$ & $\mathrm{n}-C_{7}^{0}$ & $\mathrm{n}-C_{8}^{0}$ & $\mathrm{n}-C_{9}^{0}$ & $\mathrm{n}-C_{10}^{0}$ & Total \\
\hline content $/ \%$ & 1.25 & 6.35 & 6.18 & 6.47 & 6.22 & 3.97 & 0.50 & 30.94 \\
\hline
\end{tabular}

Table S2. Properties of zeolite 5A

\begin{tabular}{lcl}
\hline \multicolumn{1}{c}{ Properties } & $\begin{array}{l}\text { Zeolite } 5 \mathrm{~A} \\
\text { before use }\end{array}$ & $\begin{array}{l}\text { Zeolite 5A } \\
\text { after use }\end{array}$ \\
\hline Bulk density, $\mathrm{kg} / \mathrm{m}^{3}$ & 820 & 821 \\
Crushing strength, $\mathrm{N}$ & 48 & 48 \\
Loss on ignition $\left(1000^{\circ} \mathrm{C}\right), \mathrm{wt} \%$ & 9.0 & 9.1 \\
Specific area, $\mathrm{m}^{2} / \mathrm{g}$ & 447.3 & 421.1 \\
Micropore average diameter, $\mathrm{nm}$ & 0.50 & 0.50 \\
Mesopore average diameter, $\mathrm{nm}$ & 7.51 & 7.89 \\
BJH pore volume, $\mathrm{cm}^{3} / \mathrm{g}$ & 0.095 & 0.091 \\
\hline
\end{tabular}


Table S3. Potential aromatic contents of the SGPC naphtha and the raffinate oil

\begin{tabular}{ccc}
\hline & SGPC naphtha & raffinate oil with CIO \\
\hline C6 cycloalkane/\% & 5.99 & 8.38 \\
C7 cycloalkane $/ \%$ & 11.59 & 16.21 \\
C8 cycloalkane/\% & 5.72 & 8.00 \\
aromatics $/ \%$ & 8.61 & 12.04 \\
\hline potential aromatic content $/ \%$ & 30.47 & 42.61 \\
\hline
\end{tabular}

Table S4. Olefin yields of the desorption oil and the SGPC naphtha

\begin{tabular}{lcc}
\hline \multicolumn{1}{c}{ cracking Feed } & ethylene yield/\% & total olefin yield/\% \\
\hline SGPC naphtha & 31.87 & 52.51 \\
desorption oil with 90\% n-paraffins & 45.59 & 64.52 \\
desorption oil with 95.18\% n-paraffins & 46.75 & 65.53 \\
desorption oil with 96.61\% n-paraffins & 46.95 & 65.71 \\
\hline
\end{tabular}

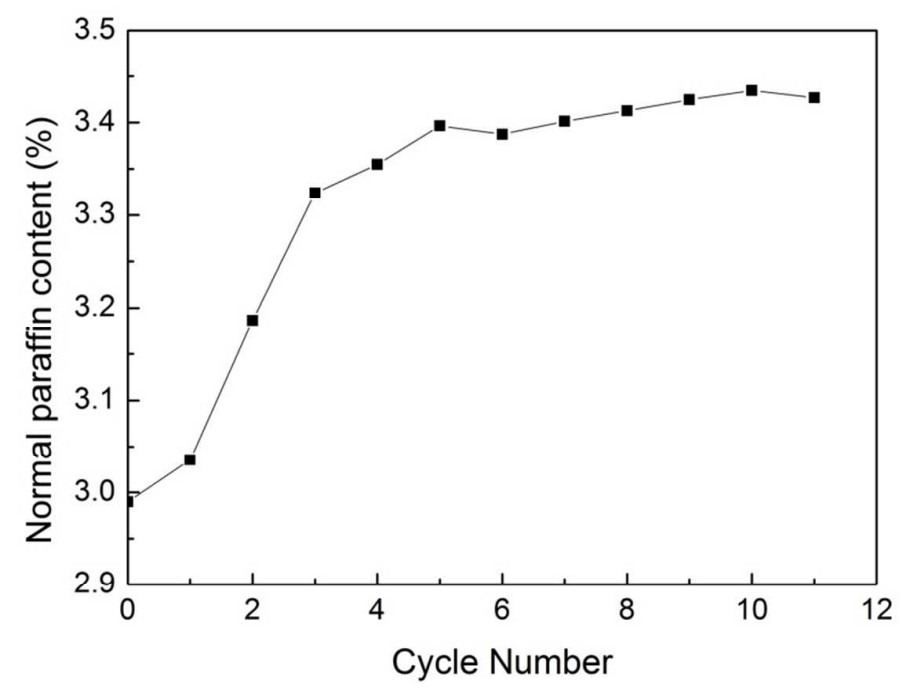

Figure S1. Normal paraffin content of raffinate oil in the CIO process 


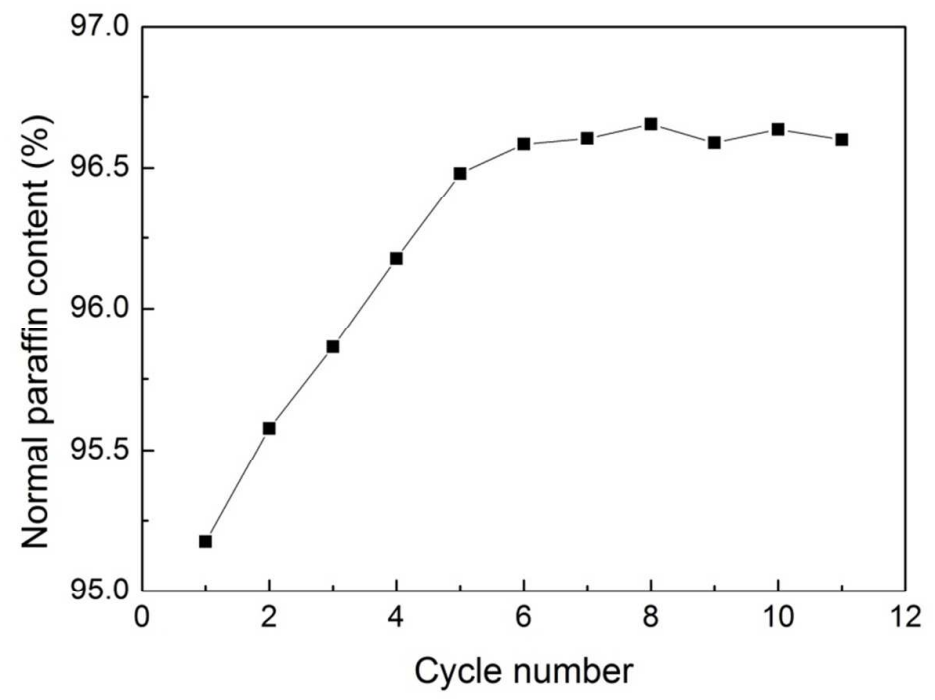

Figure S2. Normal paraffin content of desorption oil in the CIO process 